\title{
Plan, do, Study, Act Cycles, as an Alternate to Action Research for Clinically Based Inquiry
}

\author{
Bernadette Ivy Eather, Mary Elizabeth Chiarella and Judith Donoghue \\ Faculty of Health, University of Technology, Sydney, Australia
}

Received 2013-04-15, Revised 2013-09-10; Accepted 2013-09-11

\begin{abstract}
Action Research has long been the method of choice when undertaking research in clinical practice improvement. It is a method aimed at engendering ownership by the participants in order to sustain practice change. Plan, Do, Study, Act cycles (PDSA) also provide a cyclical approach to clinical practice improvement research and focus the clinical team around a specific problem. Through focus groups and discussions with staff, the contributing factors to a number of patient safety incidents were identified. A series of interventions were implemented requiring staff to intervene to mitigate patient risk and decrease patient safety incidents. These interventions were introduced utilising PDSA cycles with concomitant incremental improvements in clinical processes. Clinical practice improvement resulted in a decrease in patient safety incidents. How ever, whilst individual staff were transformed as a result of their participation in the research, the culture in which the research was conducted did not change. The elements of Action Research and PDSA will be summarised and the key similarities and differences will be compared and contrasted. The inhibiting factors to using Action Research in a dynamic acute care environment will be discussed. This study will explore how PDSA cycles, with their concurrent similarities and differences to Action Research, can provide a method for researching the implementation of a system improvement solution.
\end{abstract}

Keywords: Action Research, Patient Safety, Continuous Quality Improvement, Plan, Do, Study, Act Cycles

\section{INTRODUCTION}

Research into patient safety and clinical service quality is increasingly focusing on the contributing factors to patient risk and the systems required in order to mitigate this risk. These systems include, inter alia, clinical handover WA Health, 2012, recognition and management of deteriorating patients (ACSQHC, 2011) and medication safety programs (ACSQHC, 2011). When conducting patient safety research, it is incumbent to use effective methods in order to ensure that the development of these clinical quality programs are contextually applicable to the clinical setting and result in sustained practice change. Action Research (AR) has become the accepted method of choice when undertaking research at a clinical practice level, as it can promote ownership of practice change, resulting in greater Corresponding Author: Bernadette Ivy Eather, Faculty of Health, University of Technology, Sydney, Australia sustainability (Lazes, 2007). As a method, it has also been noteworthy in health care literature with two comprehensive systematic reviews available (Lazes, 2007; Viswanathan et al., 2004). It is a method that partners the researcher and participants in a collaborative effort in order to address issues in specific systems (Leykum et al., 2009).

However, interventions developed via AR may be difficult to translate across institutions as the emphasis is on relationships between individuals in a particular system (Leykum et al., 2009). These individuals may change over time and it can be argued that AR is more feasible if the problem is owned and solutions initiated by staff. When staff turnover is high and a problem is investigated and solutions initiated externally, as is often the case with serious patient safety incidents, the potential for effective AR is diminished. Plan, Do, Study, 
Act (PDSA) for Continuous Quality Improvement (CQI), could be considered as an alternate method for research at the clinical practice level. PDSA has a primary purpose of undertaking intervention assuming a reductionist approach by examining specific steps in the clinical practice process (Speroff and O'Connor, 2004). Arguably this method may also be transformative for the individuals involved in the process, but its primary emphasis is on clinical practice improvement, rather than an emancipatory processes. This study will demonstrate how a CQI method, with its concurrent similarities and differences to $\mathrm{AR}$, can provide a method to research system improvements.

\section{MARITALS AND METHODS}

The research in which the PDSA cycles were used was conducted following several patient safety incidents which resulted in catastrophic patient outcomes. These incidents were as a result of failure, by both medical and nursing staff, to recognise and respond to patients whose clinical conditions were deteriorating. These incidents were termed Failure to Rescue and are well described in the literature (Clarke and Aiken, 2003; Jones et al., 2011). A longitudinal series of studies was conducted in an acute ward setting in an attempt to understand the factors which led to the failure to rescue incidents occurring and to develop a range of solutions to mitigate the opportunity for recurrence of the incidents (Eather, 2010). Through focus groups and structured discussions with staff, each incident, or patient history, was utilised as a means to identify contributing factors to the incident and identify potential solutions for staff to intervene for patients at risk in order to resolve them. The contributing factors to the incidents were identified as failure to recognise, failure to respond, failure to communicate, failure to escort and failure to escalate the patient's level of risk. Collectively within this study these incidents are termed "failure to rescue".

PDSA cycles were used to design, implement and monitor specific clinical practice changes, with resulting incremental improvement. The specific clinical practice changes included the introduction of a system for the recognition and management of the deteriorating patient. A procedure was developed which included an objective scoring system to identify when a patient's clinical condition was such that intervention was essential to ensure a mitigation of patient risk. A response protocol was also developed to ensure a patient's condition could be objectively communicated and would engender a response by medical staff. The response protocol included an escalation pathway in the event that the patient's condition was such that more experienced staff were required to respond in the first instance, or if, despite a response and intervention, the patient's condition did not improve.

Follow up surveys and interviews with staff were conducted via a PDSA cycle to determine whether the research had been transformative for the staff involved through an increase in the interventions for patients at risk. The results of the follow up surveys was analysed and demonstrated a dissonance between how the staff acted in relation to the intervention for patients at risk and their beliefs in relation to how they should act. At the same time, additional failure to rescue incidents occurred and these incidents, or patient histories, were used in focus groups with staff to try and understand this dissonance.

\section{RESULTS}

The processes of clinical care were incrementally changed and improved over a period of 12 months through the introduction of a system for the recognition and management of the deteriorating patient. These changes resulted in a decrease in the identification and reporting of specific failure to rescue incidents, including failure to recognise the level of risk to the patient and failure to respond to the patient's level of risk and an increase in the intervention for patients at risk. The actions and beliefs of staff, as demonstrated in the survey, also altered over the research period, demonstrating transformation as a result of participation in the research. However, the overarching culture in which the research occurred did not alter. At the completion of the clinical practice change, the culture of the clinical environment continued to result in instances where staff did not intervene for patients at risk leading to additional (albeit different) patient safety incidents relating to aspects of failure to rescue, identified as failure to elevate patient risk to ensure an appropriate response and failure to challenge a perceived risk to patient safety.

\section{DISCUSSION}

The AR method is attributed to the pioneering work of Kurt Lewin in the 1940s. It is classicaly defined as a type of self-reflective enquiry undertaken by participants in social situations in order to improve the rationality and justice of their own practices, their understanding of these practices and the situations in which these practices are carried out (Baum et al., 2006). Lewin saw AR as a 
stepped process in which a general idea was examined, a plan developed and a decision made on the first step to take. The step is evaluated and further re-planning undertaken that may include a modification of the original idea. A decision may then be made about the next step to be taken. The cycle of planning, executing, evaluating and perhaps modifying the original idea continues until the objective is reached (McNiff, 2013). This four step process of planning, acting, observing and reflecting forms the basis of most action research (Lazes, 2007). For nurses in particular, the power of action research is the focus at the clinical practice level, which fosters a sense of ownership of the change by the clinical staff and makes change more likely to be sustained (Lazes, 2007; Marsick and Gephart, 2003). The outcomes on participants of AR include empowerment, collaboration through participation, acquisition of knowledge and social change (Glasson et al., 2008).

Despite the obvious appeal of AR, limitations in adopting this method for clinical practice improvement exist. The health care environment is not a static one and staff and processes adapt and change in response to internal and external forces. AR focuses on the relationship between individuals and the system and, in order for $\mathrm{AR}$ to exist, the project must consistently involve those responsible for practice in each of the moments of activity (Baum et al., 2006). In the clinical setting, consistent participation by staff will not always be possible as a result of workforce constraints. The high turnover of staff, via shift work, attrition or through junior medical and nursing staff rotation (Harris et al., 2002) results in variable representation in improvement projects and diminishes the feasibility of AR as a method for researching clinical practice improvement.

In addition, AR explicitly requires the participants to understand the effect of the research or intervention as part of the research process (Leykum et al., 2009). In contrast, CQI via PDSA cycles, has a primary purpose of undertaking an intervention following the identification of a problem and there is no expectation that the staff will necessarily undergo social or emancipatory change as a result of their participation. When conducting clinical research into mitigating patient risk and improving clinical quality, a method that was specifically developed for patient safety and system improvement could be a more appropriate vehicle for implementing and researching change in the clinical setting.

PDSA was first introduced by Demming in 1993 and refined by (Berwick and Nolan, 1998), as a means to provide a model by which clinical practice improvements could be made (Berwick and Nolan, 1998). It was developed following the identification of components that improve both organisational and individual performance. The components were identified as being an aim, measurement, good ideas for change and testing. Berwick and Nolan (1998) argue that improvement by definition means that something has actually changed. PDSA requires testing of real changes on a small scale and, following these small scale tests, actions adjusted according to what information is derived from these tests (Berwick and Nolan, 1998). PDSA involves an approach with repeated cycles of incremental improvement. The PDSA model for improvement attempts to strike a pragmatic balance between the need for clinical practice change to mitigate patient risk and the desire for this change to be timely and scientifically grounded. PDSA is situation specific and the intended outcomes are related to an identified problem.

The cycles in both AR and PDSA involve a combination of action and reflection or review of the action, in order to modify further actions to ensure an improvement in an identified problem (McNiff, 2013). Coupled with the cyclical nature of the method, PDSA has many features coherent with AR. PDSA also calls for the participation of key individuals who have central knowledge of the process being changed to be involved in the change process. However, it accepts that these key individuals may change and its focus is on the safety of the patient population, rather than the actual group of participants. Both methods require the focus of the clinical team on an identified problem and can be transformative for the participating individuals. This transformation is aimed at developing expertise which can be transferred to additional clinical practice issues. A summary of the similarities and differences between PDSA and AR is listed in Table 1.

This research also demonstrated that, without changing the culture in which clinical practice improvement took place, comprehensive systems to mitigate patient risk were not achieved. Both PDSA and AR could be utilised to improvement clinical practice and transform staff as a result of their participation in the research. However, several of the identified factors which contributed to patient safety incidents were attributable to the culture in which the incidents occurred. These factors were not addressed by the PDSA method and suggest that research into patient safety requires a more sophisticated approach to ensure cultural aspects of clinical care are addressed as part of any intervention. 
Bernadette Ivy Eather et al. / International Journal of Research in Nursing 4 (2): 34-39, 2013

Table 1. Similarities and Differences between Action Research and PDSA

Similarities and difference between action research and PDSA

\begin{tabular}{|c|c|c|}
\hline \multirow[b]{2}{*}{ Aim of research } & \\
\hline & Action Research & PDSA \\
\hline \multirow{19}{*}{ Involvement of staff } & Problem solving & Problem solving \\
\hline & Improving process of care & Improving process of care \\
\hline & Understanding the effect of & Emphasis on intervention \\
\hline & the research & \\
\hline & Emphasis on relationships & \\
\hline & between individuals & \\
\hline & in system & \\
\hline & Requires the participation & \\
\hline & of a team of staff with intimate & \\
\hline & knowledge of the process under & \\
\hline & improvement. Requires the same & \\
\hline & group staff to be involved & \\
\hline & throughout the research. & \\
\hline & Requires the participation of a & \\
\hline & team of staff with intimate knowledge & \\
\hline & of the process under improvement. & \\
\hline & Does not require the same staff to be & \\
\hline & involved, just that those involved & \\
\hline & have knowledge of the process. & \\
\hline \multirow[t]{29}{*}{ Iterative Steps in Method } & Plan & Plan \\
\hline & Planning is critically informed & Plan is constructive and \\
\hline & based on the identification of a & informed-based on information \\
\hline & change, or improvement to & that demonstrates a problem in \\
\hline & practice, which is considered & clinical practice. It aims \\
\hline & desirable. The plan should aim to & to test selected improvements or \\
\hline & focus on improvement. & changes. Who? What? Where? When? \\
\hline & & Testing should demonstrate \\
\hline & & a belief that a change. \\
\hline & & will result in an improvement \\
\hline & Action & Do \\
\hline & The plan, or improvement, is & Carry out the improvement \\
\hline & implemented by the group members. & or change and collect data for analysis. \\
\hline & Actioning the plan may involve & Document problems and \\
\hline & adaptation secondary to & unexpected observations \\
\hline & unforeseen effects or constraints. & to aid in understanding \\
\hline & & why a change did or did not \\
\hline & & result in an improvement. \\
\hline & Observe & Study \\
\hline & The action should be & Study the results. Has \\
\hline & observed to collect evidence & the test resulted in an \\
\hline & that allows for evaluation. & improvement? Data \\
\hline & The action process and its & analysis aims to identify \\
\hline & effects within the context & where change was well executed, \\
\hline & of the situation should be observed. & where support processes were \\
\hline & & adequate or inadequate \\
\hline & & and whether the test has \\
\hline & & resulted in an improvement. This can \\
\hline & & be carried out through group discussion \\
\hline
\end{tabular}


Table 1. Continue

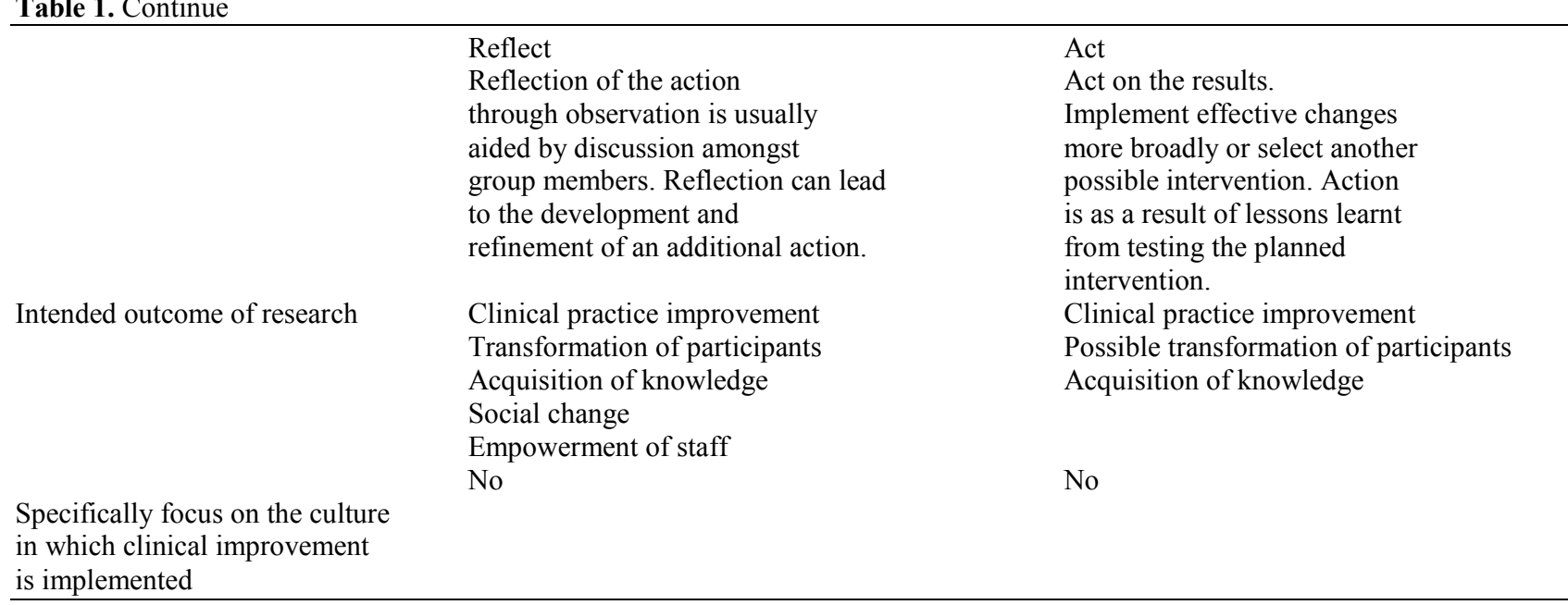

Transforming the staff who are present at the time does not necessarily result in the transformation of the clinical culture and it is the combined transformation of the clinical practice, the participants and the culture in combination which is more likely to change systems in sustained, transferrable ways and result in mitigation of patient risk.

\section{CONCLUSION}

It is the fundamental differences between $\mathrm{AR}$ and PDSA which has led to the use of PDSA for research into patient safety issues. AR is socially oriented and intends that outcomes will be evidenced through changes in social situations, systems and conditions. In contrast, PDSA is situation specific and the intended outcomes are primarily related to the identified problem. Patient safety issues can be identified, system improvement solutions developed and implementation plans directed by those external to the clinical team. This is evidenced in large scale clinical practice collaborative projects such as those for medication safety, clinical handover and the recognition and management of the deteriorating patient (ACSQHC, 2011) WA Health, 2012. The strengths of AR can be undermined when the problem is not owned and solutions not initiated by the staff involved in the research. This, coupled with a clinical environment in which staff turnover is high, has the potential for effective AR to be diminished. PDSA is effective as a method for patient safety research, where the primary purpose is the mitigation of patient risk, since it is situation specific to an intervention. PDSA can be utilised as a method to work with staff in order to implement clinical practice improvement, which is not workforce dependent. With PDSA, if the staff do turn over, then the clinical practice improvement and system changes remain in place. However, this study argues that, unless the culture of an organisation and clinical environment in which the clinical practice improvement takes place is also changed, the practice improvement will not address all the elements which contribute to patient risk.

\section{REFERENCES}

ACSQHC, 2011. National safety and Quality Service Standards. Australia.

Baum, F., C. MacDougall and D. Smith, 2006. Participatory action research. J. Epidemiol. Commun. Health, 60: 854-857. DOI: 10.1136/jech.2004.028662

Berwick, D.M. and T.W. Nolan, 1998. Physicians as leaders in improving health care: A new series in annals of internal medicine. Ann. Int. Med., 128: 289-292. PMID: 9471932

Clarke, S. and L. Aiken, 2003. Failure to rescue: Needless deaths are prime examples of the need for more nurses at the bedside. Am. J. Nurs., 103: 42-47.

Eather, B., 2010. Failure to rescue: A descriptive study of the experience of nurses in the intervention for patients at risk in the acute ward setting. University of Technology. 
Glasson, JB., E.M. Change and J.W. Bidwell, 2008. The values of participatory action research in clinical nursing practice. Int. J. Nurs. Practice, 14: 34-39. DOI: $10.1111 / \mathrm{j} .1440-172 X .2007 .00665 . x$

Harris, M., P. Gavel and W. Conn, 2002. Planning Australia's hospital workforce. Aus. Health Rev., 25: 61-77. PMID: 12474502

Jones, S., A. Bottle and P. Griffiths, 2011. An assessment of 'failure to rescue' derived from routine NHS data as a nursing sensitive patient safety indicator for surgical in patient care. King's College London.

Lazes, P., 2007. Participatory action research leading to innovation and sustained changes. Cornell University.

Leykum, L., J. Pugh, H. Lanham, J. Harmon and J.R. McDaniel, 2009. Implementation research design: Integrating participatory action research into randomized controlled trials. Implement. Sci., 4: 6969. PMID: 19852784
Marsick, V.J. and M.A. Gephart, 2003. Action research: Building the capacity for learning and change. Acad. J., 26: 14-18.

McNiff, J., 2013. Action Research: Principles and Practice. 1st Edn., Routledge, New York, ISBN-10: 0415535255, pp: 240.

Speroff, T. and G. O'Connor, 2004. Study designs for pdsa quality improvement research. Q. Manage. Health Care, 13: 17-32. PMID: 14976904

Viswanathan, M., A. Ammerman, E. Eng, G. Garlehner and K.N. Lohr, 2004. Community-based participatory research: Assessing the evidence. Evidence Report Technol. Asses., 99: 1-8. PMID: 15460504 